УДК 621.391 .82

\title{
ДЕКОМПОЗИЦИЯ КУСОЧНО-ПОЛИНОМИАЛЬНОЙ МОДЕЛИ ПРЕДЫСКАЗИТЕЛЯ ДЛЯ УСИЛИТЕЛЯ МОЩНОСТИ
}

\author{
СОЛОВЬЕВА Е. Б.
}

Санкт-Петербургский государственный электротехнический университет ЛЭТИ, Россия, Санкт-Петербург, 197376, ул. профессора Попова, д. 5

\begin{abstract}
Аннотация. Декомпозиция кусочно-полиномиальной модели предысказителя выполнена с учетом динамики изменения модуля комплексной огибающей сигнала, преобразуемого в усилителе. Декомпозиционная модель обеспечивает более высокую точность компенсации нелинейных искажений сигналов в усилителе по сравнению с кусочно-полиномиальной моделью предысказителя. Сравнительный анализ моделей предысказителей выполнен при линеаризации модели Винера-Гаммерштейна усилителя мощности
\end{abstract}

Ключевые слова: усилитель мощности; линеаризация; предыстортер; нелинейная компенсация; модель Вольтерры; нелинейный оператор

Усилитель мощности (УМ) является неотъемлемой частью многих систем связи. УМ - нелинейное устройство, в котором передаваемый сигнал искажается, его спектр расширяется и выходит за границы полосы пропускания используемого канала связи. В результате в многоканальных системах связи усиливаются искажения, вызванные влиянием соседних каналов друг на друга (межканальная интерференция) [1-3].

Для предотвращения расширения спектров выходных сигналов УМ, а также поддержания высокой энергетической эффективности усилителя (высокий КПД) выполняется линеаризация характеристик усилителя. Один из универсальных способов линеаризации цифровое предыскажение (предкомпенсация), для которого характерны робастность, простота аппаратной реализации, высокий уровень подавления нелинейности устройства [3].

Задача предкомпенсатора DPD (предысказитель, digital predistorter) - линеаризовать
УМ путем внесения предыскажения, компенсирующего нелинейные искажения усилителя мощности. В широкополосных каналах связи усилитель с высоким КПД обладает динамической нелинейностью и описывается нелинейной моделью с памятью. Следовательно, DPD - цифровое нелинейное динамическое устройство [3].

Модели DPD разнообразны: нерекурсивные и рекурсивные полиномиальные конструкции, различные типы нейронных сетей [3]. Важную роль в аппаратной реализации DPD играет простота модели предкомпенсатора, поэтому перспективным остается синтез DPD на основе модификаций усеченного ряда Вольтерры, в частности, простейшей из них - полинома с памятью [1-3]. Точность линеаризации усилителя повышается при переходе от полиномиальной модели DPD к кусочно-полиномиальной (КП) модели [4]. Входным сигналом КП-модели является векторный комплексный сигнал, состоящий из подсигналов, сфор- 